ORIGINAL ARTICLE

\title{
A study to determine plasma antioxidant concentrations in patients with Barrett's oesophagus
}

\author{
D M Clements, D A Oleesky, S C Smith, H Wheatley, D A Hullin, T J Havard, D J Bowrey
}

J Clin Pathol 2005;58:490-492. doi: 10.1136/jcp.2004.023721

See end of article for authors' affiliations

.....................

Correspondence to: Mr D J Bowrey,

Department of Surgery,

University Hospital of

Wales, Heath Park,

Cardiff CF14 4XN, UK;

bowrey@dialstart.net

Accepted for publication 24 November 2004

\begin{abstract}
Background: Dietary questionnaire studies have suggested that patients with oesophageal adenocarcinoma are deficient in antioxidants. It is not known whether the same holds true for patients with the precursor lesion, Barrett's oesophagus.

Aims: To evaluate the hypothesis that patients with Barrett's oesophagus are deficient in antioxidants compared with patients without evidence of Barrett's oesophagus.

Patients and methods: Plasma antioxidant profiles (copper, selenium, zinc; vitamins A, C, and E; carotenoids) were determined for patients with Barrett's oesophagus ( $n=36$ ), patients with erosive oesophagitis ( $n=32$ ), and patient controls ( $n=35$ ).

Results: Patients with Barrett's oesophagus had significantly lower plasma concentrations of selenium, vitamin $C, \beta$ cryptoxanthine, and xanthophyll compared with the other groups.

Conclusions: This study confirms the hypothesis that patients with Barrett's oesophagus are deficient in certain antioxidants.
\end{abstract}

A n epidemiological link between antioxidant deficiency and squamous cell carcinoma of the oesophagus has been established, most notably in China. ${ }^{12}$ Furthermore, antioxidant supplementation has been shown to produce modest reductions in the risk of progression of squamous dysplasia in this high risk population. ${ }^{3}$

"We undertook our current study to evaluate the hypothesis that patients with Barrett's oesophagus are deficient in antioxidants compared with patients with reflux who have no evidence of Barrett's oesophagus"

The role of antioxidants in adenocarcinoma of the oesophagus is unclear. The principal risk factors for this cancer subtype are gastro-oesophageal reflux disease ${ }^{4}$ and increased body mass index..$^{5-8}$ Ten studies have examined the diets of patients with oesophageal adenocarcinoma by means of structured questionnaires. ${ }^{7-16}$ Although the conclusions of these studies differed slightly, in general, patients were found to have lower intakes of antioxidants, citrus fruits, and raw vegetables compared with control populations. Two studies have examined selenium values in patients with Barrett's oesophagus and concluded that high concentrations of this antioxidant were associated with a reduced risk of progression from metaplasia to carcinoma. ${ }^{17} 18$

No study to date has compared antioxidant concentrations in patients with Barrett's oesophagus, the precursor lesion for adenocarcinoma, and lesser grades of oesophageal injury. Therefore we undertook our current study to evaluate the hypothesis that patients with Barrett's oesophagus are deficient in antioxidants compared with patients with reflux who have no evidence of Barrett's oesophagus.

\section{PATIENTS AND METHODS}

Plasma antioxidant profiles were determined for a prospectively enrolled cohort of subjects attending the endoscopy suite at the Royal Glamorgan Hospital, Llantrisant, UK, during the period May to September 2003. Our study was approved by the Bro-Taf local research ethics committee (protocol 03/5011) and the Royal Glamorgan Hospital research and development board. Each patient provided written informed consent for venepuncture.

Because antioxidants may act as reverse acute phase reactants, ${ }^{19}$ patients with occult inflammatory processes were excluded on the basis of either a raised $C$ reactive protein concentration ( $>10 \mathrm{mg} /$ litre) or hypoalbuminaemia $(<32$ g/litre). Additional exclusion criteria were the presence of gastroduodenal ulceration and a history of pancreatitis or previous foregut surgery, other than cholecystectomy.

Patients were divided into the following study groups:

(1) 36 patients (33 men, three women) with Barrett's oesophagus (defined by endoscopic columnar lined oesophagus and intestinal metaplasia on biopsy), mean age 57 years (range, 39-85);

(2) 32 patients (20 men, 12 women) with Los Angeles grade $\mathrm{B}$ or $\mathrm{C}$ erosive oesophagitis, mean age 59 years (range, 35-77);

(3) 35 patient controls ( 15 men, 20 women) free of reflux symptoms who had normal endoscopic appearances in the oesophagus, stomach, and duodenum, mean age 49 years (range, 20-72).

\section{Sample size determination}

In a previous study, we identified significant differences in the antioxidant concentrations between patients with chronic pancreatitis and healthy control subjects, ${ }^{20}$ and the magnitude of the differences was in excess of one standard deviation. For our current study, we assumed that the magnitude of the differences between Barrett's and control patients would be more modest, in the order of 0.75 standard deviations. Assuming a significance concentration of $5 \%$ and a power of $80 \%$, equal groups of 30 patients would be required.

\section{Assay details}

The biochemical parameters assessed were plasma concentrations of:

- the trace elements copper, selenium and zinc;

- vitamins $\mathrm{A}, \mathrm{C}$, and $\mathrm{E}$; 
Table 1 Trace element concentrations

\begin{tabular}{llll}
\hline Analyte & Barrett's oesophagus $(\mathbf{n}=\mathbf{3 6})$ & Erosive oesophagitis $(\mathbf{n}=\mathbf{3 2})$ & Patient controls $(\mathbf{n}=\mathbf{3 5})$ \\
\hline Copper $(\mu \mathrm{mol} / \mathrm{l})$ & $16.0(15.0$ to 16.9$)$ & $16.9(15.5$ to 18.3$)$ & $16.3(14.9$ to 17.7$)$ \\
Selenium $(\mu \mathrm{mol} / \mathrm{l})$ & $0.72^{*}(0.67$ to 0.78$)$ & $0.75(0.71$ to 0.79$)$ & $0.81(0.74$ to 0.87$)$ \\
Zinc $(\mu \mathrm{mol} / \mathrm{l})$ & $13.3(11.4$ to 15.1$)$ & $12.9(12.2$ to 13.5$)$ & $13.1(11.6$ to 14.6$)$ \\
\hline
\end{tabular}

Values shown are mean (95\% confidence interval).

${ }^{*} \mathrm{p}=0.05 v$ patient controls.

Table 2 Vitamin concentrations

\begin{tabular}{llll}
\hline Analyte & Barrett's oesophagus $(\mathbf{n = 3 6 )}$ & Erosive oesophagitis (n=32) & Patient controls (n=35) \\
\hline Vitamin A $(\mu \mathrm{mol} / \mathrm{l})$ & $2.17(1.97$ to 2.38$)$ & $2.01(1.82$ to 2.21$)$ & $1.97(1.83$ to 2.11$)$ \\
Vitamin $C(\mu \mathrm{mol} / \mathrm{l})$ & $18.3^{*}(13.2$ to 23.4$)$ & $26.6(19.6$ to 33.6$)$ & $27.1(21.4$ to 32.7$)$ \\
Vitamin $E(\mu \mathrm{mol} / \mathrm{l})$ & $26.7(23.0$ to 30.4$)$ & $29.1(25.6$ to 32.6$)$ & $28.3(25.1$ to 31.4$)$ \\
\hline
\end{tabular}

Values shown are mean ( $95 \%$ confidence interval).

${ }^{*} p=0.02 v$ patient controls and $p=0.05 v$ erosive oesophagitis.

- the carotenoids ( $\alpha$ carotene, $\beta$ carotene, $\beta$ cryptoxanthine, lycopene, and xanthophyll).

Venous blood was collected from each subject after a six hour fast (immediately before endoscopy).

Samples for trace element analysis were collected in trace element free sodium heparin Vacutainers (Becton Dickinson, Le Pont de Claix, France); those for vitamin analysis were taken into lithium heparin Vacutainers (Becton Dickinson) and transported to the laboratory in a light excluding container. All samples were centrifuged within 30 minutes of collection; separated plasma was divided into aliquots and stored in $\mathrm{a}-70^{\circ} \mathrm{C}$ freezer.

Samples were subsequently analysed in batches in the medical biochemistry department, University Hospital of Wales, Cardiff, UK. Copper and zinc were measured using flame atomic absorption on an FS-220 spectrophotometer (Varian Inc, Lexington, Massachusetts, USA). Selenium was measured by electrothermal graphite furnace atomic absorption using a palladium nitrate matrix modifier on an AA-600 spectrophotometer (Varian Inc). Vitamins A, C, and E and the carotenoids were measured by means of high performance liquid chromatography with spectrophotometric detection.

Continuous data were compared using the unpaired $t$ test, with significance assumed at the $5 \%$ level.

\section{RESULTS}

Tables 1-3 summarise the antioxidant profiles. Patients with Barrett's oesophagus had significantly lower plasma concentrations of selenium, vitamin $C, \beta$ cryptoxanthine, and xanthophyll compared with the other groups.

\section{DISCUSSION}

It is unclear why some patients with gastro-oesophageal reflux disease develop Barrett's oesophagus. Physiological oesophageal studies have shown that Barrett's oesophagus is closely linked to poor lower oesophageal barrier function, hiatus hernia, and high levels of oesophageal acid (and bile) exposure. ${ }^{21}$ Nonetheless, even for similar oesophageal reflux exposure, end organ manifestations vary between individuals; genetic factors may be implicated. Our current study evaluated whether or not antioxidants could influence the development of Barrett's oesophagus.

There are few publications on antioxidants in patients with Barrett's oesophagus. However, several studies have evaluated antioxidant intakes in patients with oesophageal adenocarcinoma using dietary questionnaires. ${ }^{7-16}$ Dietary inventories were completed by patients and antioxidant intake calculated based upon the known content of each food substance. These reports suggested a protective effect for diets rich in citrus fruits, certain vegetables, and several antioxidants, notably $\beta$ carotene and vitamin C.

Two studies from the same institution of patients with Barrett's oesophagus identified low selenium concentrations as a risk factor for progression to adenocarcinoma. ${ }^{17}$ Rudolph et al evaluated selenium concentrations in patients with Barrett's oesophagus enrolled in a surveillance programme. ${ }^{18}$ They found that low selenium concentrations were associated with an increased risk of progression to high grade dysplasia, loss of wild-type p53, and aneuploidy. No association was seen between low selenium concentrations and loss of the wild-type pl6 gene, an early event in carcinogenesis, leading the authors to speculate that low selenium concentrations are a late event in the development of oesophageal adenocarcinoma.

A recent Cochrane review ${ }^{22}$ examining the potential role of antioxidant supplementation in the prevention of gastrointestinal malignancy found no evidence of a protective effect, with the exception of a modest benefit for selenium. It should be stressed that the study groups were heterogeneous and included patients with hepatocellular, colorectal, oesophageal, gastric, and pancreatic cancers.

\section{"Antioxidants are central to the cellular defence mechan- ism against oxidative damage"}

Our current study has taken the investigation one step further back in an attempt to elucidate potential differences in antioxidant concentrations between patients with reflux, with and without Barrett's oesophagus. Antioxidants are

\section{Take home messages}

- Patients with Barrett's oesophagus were deficient in certain antioxidants - they had significantly lower plasma concentrations of selenium, vitamin C, $\beta$ cryptoxanthine, and xanthophyll than the other groups

- It is possible that antioxidant supplementation could reduce the mortality risk for glandular dysplasia and adenocarcinoma in these patients and appropriate studies are needed to explore this issue 
Table 3 Carotenoid concentrations

\begin{tabular}{llll}
\hline & Barrett's oesophagus $(\mathbf{n}=\mathbf{3 6})$ & Erosive oesophagitis $(\mathbf{n}=\mathbf{3 2})$ & Patient controls $(\mathbf{n}=\mathbf{3 5})$ \\
\hline$\alpha$ Carotene $(\mu \mathrm{mol} / \mathrm{l})$ & $0.05(0.04-0.06)$ & $0.05(0.04-0.06)$ & $0.05(0.04-0.06)$ \\
$\beta$ Carotene $(\mu \mathrm{mol} / \mathrm{l})$ & $0.28(0.24-0.32)$ & $0.30(0.26-0.34)$ & $0.32(0.27-0.37)$ \\
$\beta$ Cryptoxanthine $(\mu \mathrm{mol} / \mathrm{l})$ & $0.06^{*}(0.05-0.08)$ & $0.10(0.08-0.13)$ & $0.08(0.07-0.10)$ \\
Lycopene $(\mu \mathrm{mol} / \mathrm{l})$ & $0.32(0.26-0.38)$ & $0.28(0.20-0.35)$ & $0.26(0.21-0.31)$ \\
Xanthophyll $(\mu \mathrm{mol} / \mathrm{l})$ & $0.35^{* *}(0.27-0.44)$ & $0.55(0.41-0.69)$ & $0.48(0.33-0.63)$ \\
\hline Values shown are mean $(95 \%$ confidence interval). & & \\
${ }^{*} \mathrm{p}=0.008$ v oesophagitis; ${ }^{* *} \mathrm{p}=0.002$ v oesophagitis. & & \\
\hline
\end{tabular}

central to the cellular defence mechanism against oxidative damage-they catalyse the breakdown of hydrogen peroxide and fatty acyl lipid peroxides, in the presence of reduced glutathione, to water and the corresponding alcohols. Such peroxides are a source of potentially damaging free radicals, which can cause peroxidation of polyunsaturated fatty acids in the cell membrane. The toxic oxygen derivatives, which are neutralised by the antioxidants, are produced by normal cellular metabolic activity and by a variety of injurious agents. ${ }^{23}$

Our main findings were that patients with Barrett's oesophagus had significantly lower plasma concentrations of the antioxidants selenium, vitamin $C, \beta$ cryptoxanthine, and xanthophyll compared with patient controls. What is the therapeutic potential of these observations? More information is available on squamous dysplasia and neoplasia, notably from China. The epidemiology of squamous carcinoma in high incidence regions of China (Linxian province) supports an association with antioxidant deficiency. ${ }^{12}$ Large scale antioxidant supplementation studies in this region revealed modest reductions in the mortality risk from oesophageal or gastric cardia malignancy. ${ }^{3}$ It is an appealing concept that the same will hold true for glandular dysplasia and adenocarcinoma.

\section{Authors' affiliations}

D M Clements, T J Havard, D J Bowrey, Surgery Department, Royal Glamorgan Hospital, Llantrisant CF72 8XR, UK

D A Oleesky, S C Smith, H Wheatley, Medical Biochemistry Department, University Hospital of Wales, Cardiff CF14 4XW, UK

D A Hullin, Biochemistry Department, Royal Glamorgan Hospital

\section{REFERENCES}

1 Nomura AM, Ziegler RG, Stemmermann GN, et al. Serum micronutrients and upper aerodigestive tract cancer. Cancer Epidemiol Biomarkers Prev 1997;6:407-12.

2 Hu J, Nyren O, Wolk A, et al. Risk factors for esophageal cancer in northeast China. Int J Cancer 1994:57:38-46.

3 Dawsey SM, Wang GQ, Taylor PR, et al. Effects of vitamin/mineral supplementation on the prevalence of histological dysplasia and early cancer of the esophagus and stomach: results from the dysplasia trial in Linxian, China. Cancer Epidemiol Biomarkers Prev 1994;3:167-72.
4 Lagergren J, Bergstrom R, Lindgren A, et al. Symptomatic gastroesophageal reflux as a risk factor for esophageal adenocarcinoma. N Engl J Med 1999:340:825-31.

5 Lagergren J, Bergstrom R, Nyren $\mathrm{O}$. Association between body mass index and adenocarcinoma of the esophagus and gastric cardia. Ann Intern Med 1999;130:883-90.

6 Tretli S, Robsahm TE. Height, weight and cancer of the esophagus and stomach: a follow-up study in Norway. Eur J Cancer Prev 1999;8:1 15-22.

7 Brown LM, Swanson CA, Gridley G, et al. Adenocarcinoma of the esophagus: role of obesity and diet. J Natl Cancer Inst 1995:87:104-9.

8 Cheng KK, Sharp L, McKinney PA, et al. A case-control study of esophageal adenocarcinoma in women: a preventable disease. $\mathrm{Br} J$ Cancer 2000;83:127-32.

9 Kabat GC, Ng SK, Wynder EL. Tobacco, alcohol intake, and diet in relation to adenocarcinoma of the esophagus and gastric cardia. Cancer Causes Control 1993;4:123-32.

10 Palli D, Bianchi S, Decarli A, et al. A case-control study of cancers of the gastric cardia in Italy. Br J Cancer 1992;65:263-6.

11 Terry $\mathbf{P}$, Lagergren J, Ye W, et al. Antioxidants and cancers of the esophagus and gastric cardia. Int J Cancer 2000;87:750-4.

12 Tzonou A, Lipworth L, Garidou A, et al. Diet and risk of esophageal cancer by histologic type in a low-risk population. Int J Cancer 1996;68:300-4.

13 Chen H, Ward MH, Graubard BI, et al. Dietary patterns and adenocarcinoma of the esophagus and distal stomach. Am J Clin Nutr 2002;75:137-44.

14 Zhang ZF, Kurtz RC, Yu GP, et al. Adenocarcinomas of the esophagus and gastric cardia: the role of diet. Nutr Cancer 1997;27:298-309.

15 Mayne ST, Risch HA, Dubrow R, et al. Nutrient intake and risk of subtypes of esophageal and gastric cancer. Cancer Epidemiol Biomarkers Prev 2001; 10:1055-62.

16 Terry $\mathbf{P}$, Lagergren J, Hansen $\mathrm{H}$, et al. Fruit and vegetable consumption in the prevention of esophageal and cardia cancers. Eur J Cancer Prev $2001 ; 10: 365-9$.

17 Moe GL, Kristal AR, Levine DS, et al. Waist-to-hip ratio, weight gain, and dietary and serum selenium are associated with DNA content flow cytometry in Barrett's esophagus. Nutr Cancer 2000;36:7-13.

18 Rudolph RE, Vaughan TL, Kristal AR, et al. Serum selenium levels in relation to markers of neoplastic progression among persons with Barrett's esophagus. J Natl Cancer Inst 2003;95:750-7.

19 Hawker FH, Stewart PM, Smith PJ. Effects of acute illness on selenium status. Crit Care Med 1990;18:442-6.

20 Morris-Stiff GJ, Bowrey DJ, Davies M, et al. The antioxidant profiles of patients with recurrent acute pancreatitis and chronic pancreatitis. Am J Gastroenterol 1999:94:2135-40.

21 Cameron AJ. Barrett's esophagus. In: Castell DO, Richter JE, eds. The esophagus, 3rd ed. Philadelphia: Lippincott Williams \& Wilkins, 1999

22 Bjelakovic G, Nikolova D, Simonetti RG, et al. Antioxidant supplements for preventing gastrointestinal cancers. The Cochrane Database of Systematic Reviews, 2004, Issue 4.

23 Bowrey DJ, Morris-Stiff GJ, Puntis MCA. Selenium deficiency and chronic pancreatitis: disease mechanism and potential for therapy. HPB Surg $1999 ; 11: 207-16$. 